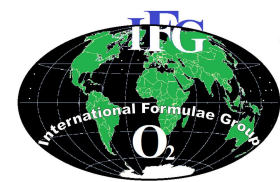

Available online at http://ajol.info/index.php/ijbcs

Int. J. Biol. Chem. Sci. 9(1): 200-216, February 2015

International Journal

of Biological and

Chemical Sciences

ISSN 1997-342X (Online), ISSN 1991-8631 (Print)

Original Paper

http://indexmedicus.afro.who.int

\title{
Pentaclethra macrophylla Benth. dans la forêt communautaire de Payo (Est- Cameroun): inventaire, productivité et commercialisation
}

\author{
Mama MOUAMFON ${ }^{1 *}$, Nicole Marie GUEDJE ${ }^{2}$, Issofa PEPAINYIENE ${ }^{3}$, \\ Louis ZAPFACK ${ }^{3}$, Jules Romain NGUEGUIM ${ }^{4}$ et Jean LEJOLY ${ }^{5}$ \\ ${ }^{1}$ Ecole Régionale Postuniversitaire d'Aménagement et de Gestion Intégrés des Forêts et \\ Territoires Tropicaux (ERAIFT),Université de Kinshasa, BP 15373, RDC. \\ ${ }^{2}$ Département de Pharmacognosie et de Chimie Pharmaceutique, Faculté de Médecine et \\ des Sciences Biomédicales, Université de Yaoundé 1, BP 25645 Yaoundé, Cameroun. \\ ${ }^{3}$ Facultés des sciences, Université de Yaoundé 1, BP 337 Yaoundé, Cameroun. \\ ${ }^{4}$ Institut de la Recherche Agricole pour le Developpement. BP 219 Kribi, Cameroun \\ ${ }^{5}$ Université Libre de Bruxelles, Laboratoire de Phytotaxonomie et Systématique Végétale, C.P. 169, \\ Avenue F. Roosevelt, 50. 1050 Bruxelles, Belgique. \\ *Auteur correspondant, E-mail : mouamfon_m@yahoo.fr; BP 12763, Yaoundé, Cameroun ; \\ Tél : (+237) $99664359 / 76302656$.
}

\section{RESUME}

Cette étude a été conduite à l'Est du Cameroun, dans la forêt communautaire de Payo. Elle a pour objet d'évaluer l'importance écologique, productive et socioéconomique de $P$. macrophylla. Le dispositif d'inventaire est constitué de quatre transects de $40 \mathrm{~m}$ x $2000 \mathrm{~m}^{2}$ (8 ha chacun) avec un taux de sondage de $2 \%$. Toutes les tiges de diamètre à hauteur de poitrine $\geq 10 \mathrm{~cm}$ ont été marquées, géoreferencées, comptées et leur diamètre mesurés à la hauteur $1,30 \mathrm{~cm}$. Les observations et entretiens, ainsi que le suivi de la filière de commercialisation ont été réalisés à Payo puis dans la ville de Lomié. L'espèce est abondante dans les forêts secondaires jeunes avec une densité de 6 tiges/ha. Le diamètre moyen et la surface terrière des tiges recencées sont respectivement estimés à 56,91 $\pm 14 \mathrm{~cm}$ et $19,46 \mathrm{~m}^{2} / \mathrm{ha}$. L'indice de régénération naturelle est évalué à 4,8 et influencé par l'intensité lumineuse qui parvient dans le sous bois. La production de 35 arbres pour une saison de fructification est estimée à 5157 graines soit en moyenne 147,34 graines/arbre. La production annuelle est estimée à 1261255 graines équivalant à 11571 combo (unité de mesure locale). La valeur marchande de ces graines est estimée à 5785572 Frs CFA (11836 \$US). L'exploitation des graines de P. macrophylla représente ainsi de bonnes perspectives de revenus pour cette localité.

(C) 2015 International Formulae Group. All rights reserved.

Mots clés : Production, commercialisation, Pentaclethra macrophylla, forêt communautaire.

\section{INTRODUCTION}

Les forêts tropicales humides renferment de nombreuses ressources en produits et services qui contribuent d'une manière générale au bien-être des populations (Betti et Lejoly, 2010; Priso et al., 2011; Ngueguim, 2013). Cependant, le couvert végétal ainsi que le patrimoine des espèces 
pourvoyeuses des produits forestiers non ligneux (PFNL) se dégradent progressivement, eu égard notamment à leur utilisation non rationnelle. Dans le bassin du Congo, le taux annuel de déforestation brute a été estimé à 0,13\% pour la période 1990-2000 et ce taux a doublé pour la période 2000-2005 (Ernst et al., 2012).

Pendant longtemps, les populations des zones forestières ont fondé beaucoup d'espoir sur l'exploitation artisanale du bois d'œuvre au détriment d'autres ressources forestières non ligneuses qui contribuent pourtant siginficativement à combler au quotidien leurs besoins de subsistance. La qualité des ressources ligneuses dans les forêts communautaires ainsi que leur gestion constituent un handicap majeur pour la mise en valeur de ces forêts; les rendements sont encore loin des prévisions établies lors du lancement du concept (Mouamfon et al., 2010). Au fil des ans, le developpement de l'économie marchande et les réformes des lois ont complexifié les procédures d'accès au bois pour les communautés rurales. Conscientes donc des difficultés liées à l'exploitation du bois et ne disposant pas d'assez de moyens pour subvenir à leurs besoins quotidiens, les populations des zones forestières en général et celles de Payo en particulier, s'intéressent davantage à l'exploitation et à la valorisation des produits forestiers non ligneux parfois peu connus à l'instar des graines de Pentaclethra macrophylla Benth. Ces graines sont exportées vers le Nigéria pour la consommation alimentaire. L'intérêt particulier pour ces graines émane de leur qualité organoleptique, du statut de l'espèce qui est non menacée et de ce qu'elle représente un produit économiquement émergent (Mandeng, 2009). Ces dernières années, on peut noter une intense activité d'exploitation locale de $P$. macrophylla ce qui suscite beaucoup d'inquiétudes quant à sa pérennité et sa disponibilité pour assurer sur le long terme son maintien et une gestion durable. Des études sont en cours sur le plan de la domestication afin d'anticiper sur le problème. L'on peut citer les travaux de Tsobeng et al. (2013). Ce contexte justifie l'intérêt de cette étude qui porte sur l'inventaire, la productivité et la commercialisation de $P$. macrophylla dans la forêt communautaire (FC) de Payo. De telles études spécifiques, localement circonscrites, permettent une meilleure connaissance des ressources et une évaluation de leur potentiel de gestion (Guedje et Bigombe, 2010 ; Guedje et al., 2010). L'une des principales préoccupations actuelles est de savoir si la capacité de production et le potentiel de régénération de $P$. macrophylla dans cette forêt sont de nature à garantir la pérennité de l'espèce. En d'autres termes : quel peut être le potentiel disponible de l'espèce dans cette forêt, en nombre de tiges à l'hectare et/ou en quantité de graines produites annuellement? Quelle est l'importance socioéconomique de cette espèce ? L'étude a donc pour objectifs d'établir la distribution de P. macrophylla dans différents types d'habitats et caractériser la production fruitière et son importance socioéconomique dans la forêt communautaire de Payo.

\section{MATERIEL ET METHODES Site d'étude}

La forêt communautaire de Payo est située en zone de forêt dense humide à environ $13 \mathrm{~km}$ de la ville de Lomié, Arrondissement de Lomié, Département du Haut-Nyong, Région de 1'Est Cameroun. Cette forêt est comprise entre $06^{\circ} 42,3^{\prime}$ et $10^{\circ} 44,7^{\prime}$ de latitude Nord ; 41 $43,2^{\prime}$, et $42^{\circ} 57,0^{\prime}$ de longitude Est, et couvre une superficie de 1600 ha. Au sens de la loi de 1994 portant régime des forêts, de la faune et de la pêche en son article 37 , alinéa 5 et aux articles 3 (11) et (16), puis 27 (4) et (5) du décret du 23 août 1995 fixant les modalités d'application du régime des forêts, « une forêt communautaire est une zone du domaine 
forestier non permanent, pouvant mesurer jusqu'à 5000 hectares, et faisant l'objet d'une convention de gestion entre une communauté villageoise et l'administration des forêts ».

Le climat est de type équatorial guinéen (chaud et humide) de régime bimodal subdivisé en 4 saisons : deux saisons de pluies (une grande et une petite) et deux saisons sèches (une grande et une petite). Cet atout climatique offre l'opportunité de deux saisons de récolte pour l'espèce étudiée. La température moyenne annuelle est de $23,4{ }^{\circ} \mathrm{C}$ (Fokou, 2008) et la moyenne annuelle des précipitations se situe entre $1600 \mathrm{~mm}$ et 1700 mm d'eau.

\section{Matériel végétal}

$P$. macrophylla, est communément appelé arbre à semelles ou Acacia du Congo, «Mubala» ou «Ebaye ». Dans la zone d'étude, l'espèce est appélée «Balaka ». Elle appartient à la famille des FabaceaeMimosoideae. Elle est endémique des plaines humides et subhumides d'Afrique de l'Ouest. $\mathrm{Au}$ Cameroun, elle est présente partout en forêt sempervirente (où elle est abondante dans les zones de Mamfé et du Dja) et en forêt semi-caducifoliée où elle est dispersée et peu grégaire (Vivien et Faure, 1996). L'espèce est présente au niveau des altitudes allant jusqu'à 500 mètres bien que la croissance peut être bonne à des altitudes plus élevées lorsque la pluviométrie est adéquate et les températures inférieures à $18{ }^{\circ} \mathrm{C}$ (Oboh, 2007). Elle peut atteindre jusqu'à $30 \mathrm{~m}$ de hauteur et $80 \mathrm{~cm}$ de diamètre. Les feuilles sont composées, elles ont entre 20 à $45 \mathrm{~cm}$ de longueur avec 10 à 12 paires de pennes opposées et longue de 7 à 13 $\mathrm{cm}$. Les fruits s'ouvrent par explosion et libèrent entre 6 et 10 graines plates pouvant atteindre $7 \mathrm{~cm}$ de longueur (Figure 1).

\section{Inventaire des pieds de $\boldsymbol{P}$. macrophylla}

Le dispositif d'inventaire s'inspire des méthodologies d'étude des produits forestiers non ligneux développées par Hall et Bawa
(1993) et reprises par Guedje (2002). Il est constitué de quatre transects de $2 \mathrm{~km}$ de longueur et $40 \mathrm{~m}$ de largeur chacun, soit une superficie totale de 32 hectares (Figure 2). Les transects implantés de part et d'autres de la route reliant Lomié à Messok, sont constitués d'une succession continue des placettes élémentaires de $50 \times 40 \mathrm{~m}^{2}$ permettant de caracteriser les types d'habitat. Le long de chaque transect et à l'intérieur des placettes, les types d'habitat (TH) sont identifiés. Il s'agit des forêts secondaires agées (FSA) caractérisées par un sous bois eclairé, la présence des grands arbres héliophiles comme Ceiba pentandra (L.) Gaertn., Triplochyton scleroxylon K. Schum. et Ricinodendron heudelotii (Baill.) Pierre ex Baill.; forêts secondaires jeunes (FSJ) caractérisées par un sous bois touffu, la présence des arbres pionniers comme Musanga cecropioides R.Br.ex Tedlie; des jachères avec une dominance des herbacées et des champs. Tous les pieds de P. macrophylla de diamètre à hauteur de poitrine (Dhp $\geq 10 \mathrm{~cm}$ ) sont identifiés, inventoriés, , leurs coordonnées GPS enregistrées et leur circonférence mesurée à l'aide d'un mètre ruban. Les caracteristiques dendrométriques estimées sont: la surface de couverture

$$
\mathrm{G}=\frac{\pi}{4} \sum_{i=1}^{n} \mathrm{D}_{\mathrm{i}}^{2}=\frac{1}{4 \pi} \sum_{i=1}^{n} \mathrm{C}_{\mathrm{i}}^{2},
$$

où $\mathrm{Di}$ est le diamètre des tiges et $\mathrm{Ci}$ la circonférence; la fréquence et le diamètre moyen Dmoyen $=\sum_{\mathrm{i}=1}^{\mathrm{n}} \frac{\mathrm{Dhpi}}{\mathrm{n}}$.

\section{Inventaire des plantules}

Le dispositif est constitué de quatre transects de $50 \mathrm{~m}$ de longueur sur $4 \mathrm{~m}$ de large placés à partir de la base de l'arbre pour un ensemble de 35 arbres (Figure 3). Les transects sont installés suivant les directions des points cardinaux pour la collecte des données sur les plantules (individus de diamètre strictement inférieur à $10 \mathrm{~cm}$ ) et des graines trouvées au sol. Ce dispositif s'inspire 
de celui de Nyiramana (2012) qui etudie la survie des graines et plantules de Carapa grandiflora dans quatre parcelles sous les couronnes de 15 arbres. Les pieds isolés de $P$. macrophylla ont été identifiés par type d'habitat et dans les différentes classes de diamètre. Puis, sous chaque pied, les jeunes plants sont inventoriés dans les différents types d'habitat et suivant les directions des quatre points cardinaux et au-delà du diamètre de l'houppier. Les caractéristiques de la plantule prises en compte concernent: la hauteur et les distances par rapport au pied de l'arbre identifié. Ces distances ont été aussi bien évaluées pour les plants que pour les graines trouvées au sol.

\section{Evaluation de la production des graines}

La méthode consiste à collecter puis compter à la fin de chaque saison de production (Février) et pendant 02 campagnes (février 2013 et février 2014) toutes les gousses de trente cinq (35) arbres matures sélectionnés dans les transects établis, distants d'au moins $50 \mathrm{~m}$ et appartenant à différentes classe de diamètre. Sous chaque pied sélectionné sont rassemblés au même endroit tous les péricarpes ou coques qui se trouvent au sol dans les environs de l'arbre en éliminant ceux des années antérieures (visiblement détériorés). Le comptage des graines par le moyen des coques est esperimenté avec succès par Nyiramana (2012). La méthode utilisée prend en compte le facteur des rongeurs afin de réduire les marges d'erreurs par comptage direct sur les graines au sol. Il a été noté dans les travaux de Forget (1996) et Nyiramana (2012) sur Carapa $s p$ que la prise en compte du facteur de déplacement des graines par les rongeurs est importante pour l'évaluation de la productivité de ce type d'espèce. Les péricarpes n'étant pas prisés par les animaux moins encore les hommes, ne subissent pas de déplacement significatif. L'on mesure la longueur de chaque péricarpe, compte le nombre de cicatrices des graines présentes sur ces derniers. Le nombre total est divisé par 2 puisque deux péricarpes au sol sont issus d'une gousse divisée en deux parties égales. On s'est aussi rassuré que les couronnes des arbres à évaluer ne soient pas traumatisées. Une équipe constituée d'au moins cinq personnes avait pour tâche de s'assurer que toutes les gousses tombées ont été bien collectées et rassemblées sous le pied sélectionné. Les moyennes (m) des gousses, des graines par gousse et par tige sont obtenues en faisant la somme arithmétique de l'effectif (Xi) de chaque paramètre et inversement proportionnelle à l'effectif total

$$
\mathrm{N}: \mathrm{m}=\frac{1}{N} \sum_{i=1}^{n} \mathrm{X}_{\mathrm{i}}
$$

\section{Suivi de l'exploitation des graines}

Un suivi a été fait auprès de 98 personnes dans l'exercice de leur activité à l'aide de fiches de suivi permettant de quantifier les collectes, estimer les revenus issus de la vente, apprécier l'intensité et la fréquence des récoltes. Cette approche a été experimenté par Guedje (2002) pour le suivi de l'exploitation de Garcinia lucida Vesque. Le critère de sélection de ces personnes était l'exercice de manière régulière ou saisonnière des activités d'exploitation et de commercialisation des graines de $P$. macrophylla.

\section{Analyse des données}

Les données issues des inventaires de $P$. macrophylla ont été traitées par les logiciels Quantum GIS 1.80 et le tableur EXCEL pour les calculs. Les paramètres considérés sont : les fréquences, moyennes et sommes des variables mesurées, la variance et l'écart-type. Les questionnaires conçus ont été codés, encodés et dépouillés à l'aide du logiciel SPSS V20 X86. 
M. MOUAMFON et al. / Int. J. Biol. Chem. Sci. 9(1): 200-216, 2015

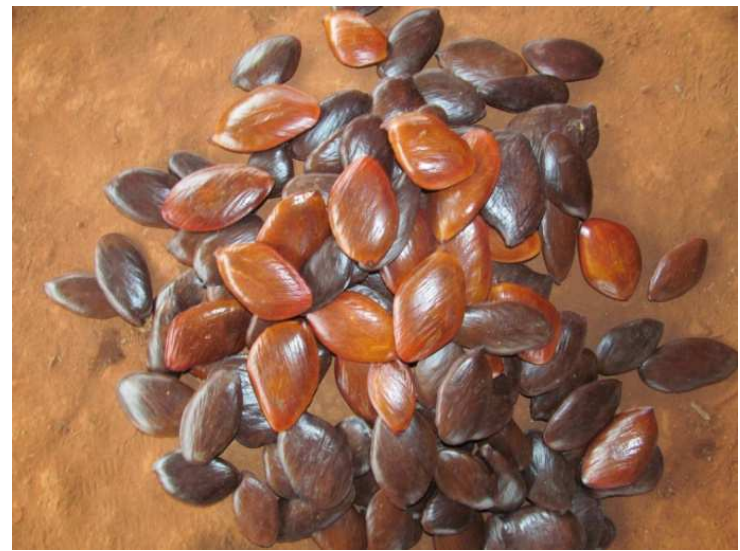

Figure 1 : Graines de P. macrophylla.

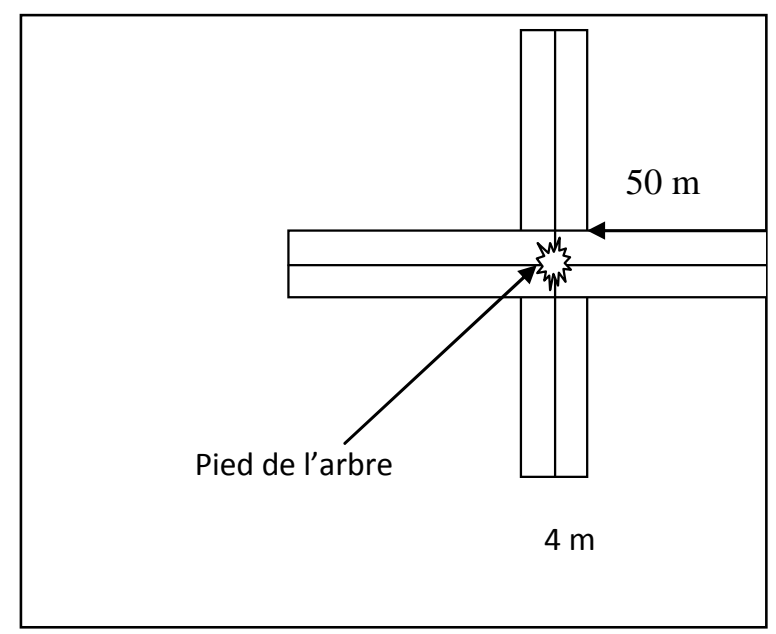

Figure 2 : Dispositif d'inventaire des populations de $P$ macrophylla.

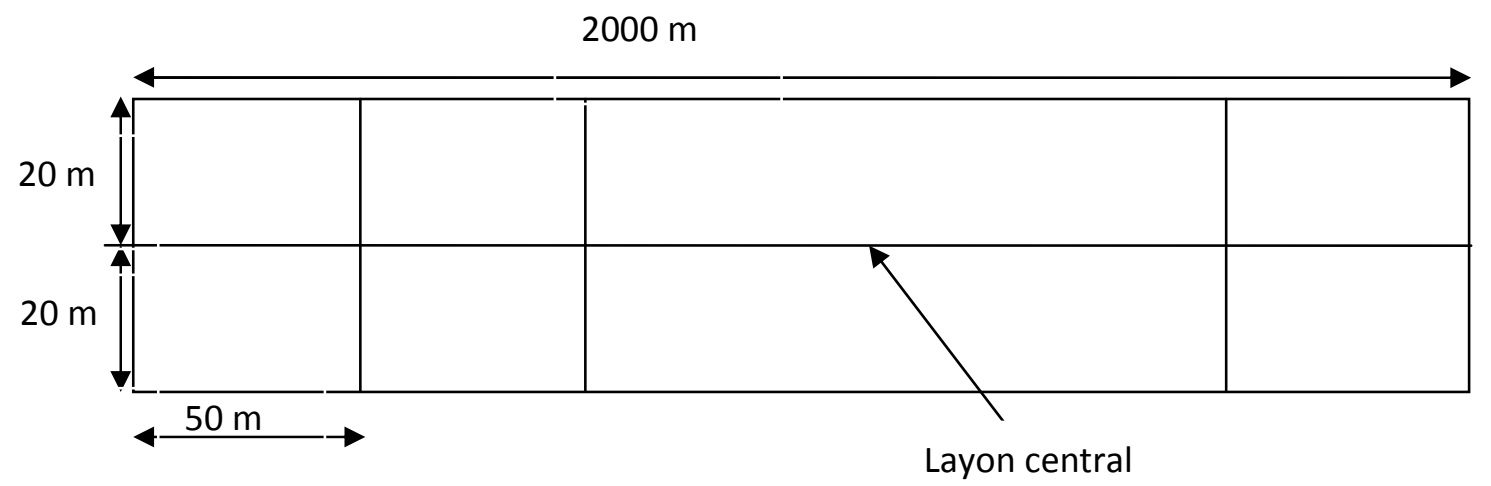

Figure 3: Dispositif d'inventaire de régénération de $P$. macrophylla. 


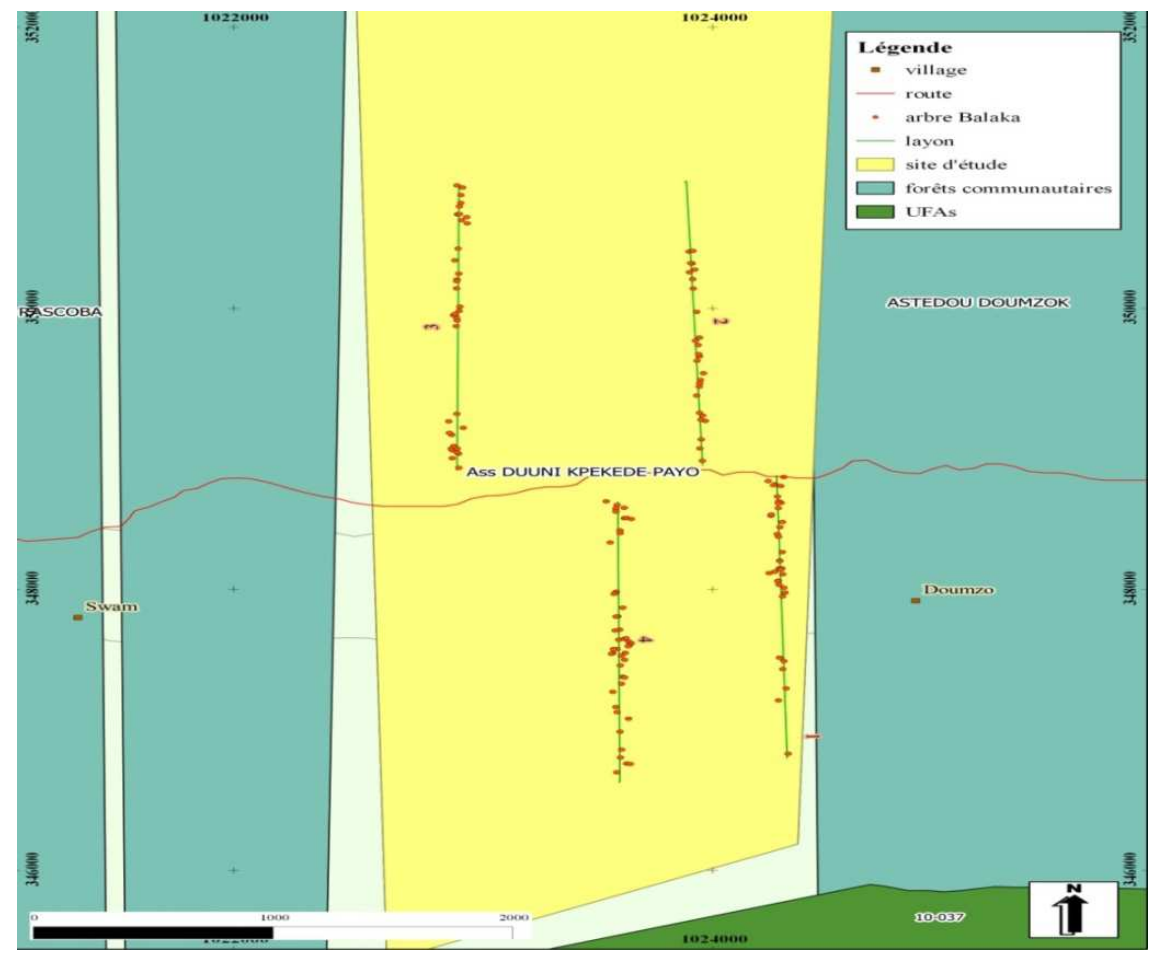

Figure 4 : Distribution de $P$. macrophylla sur les transects du site d'étude.

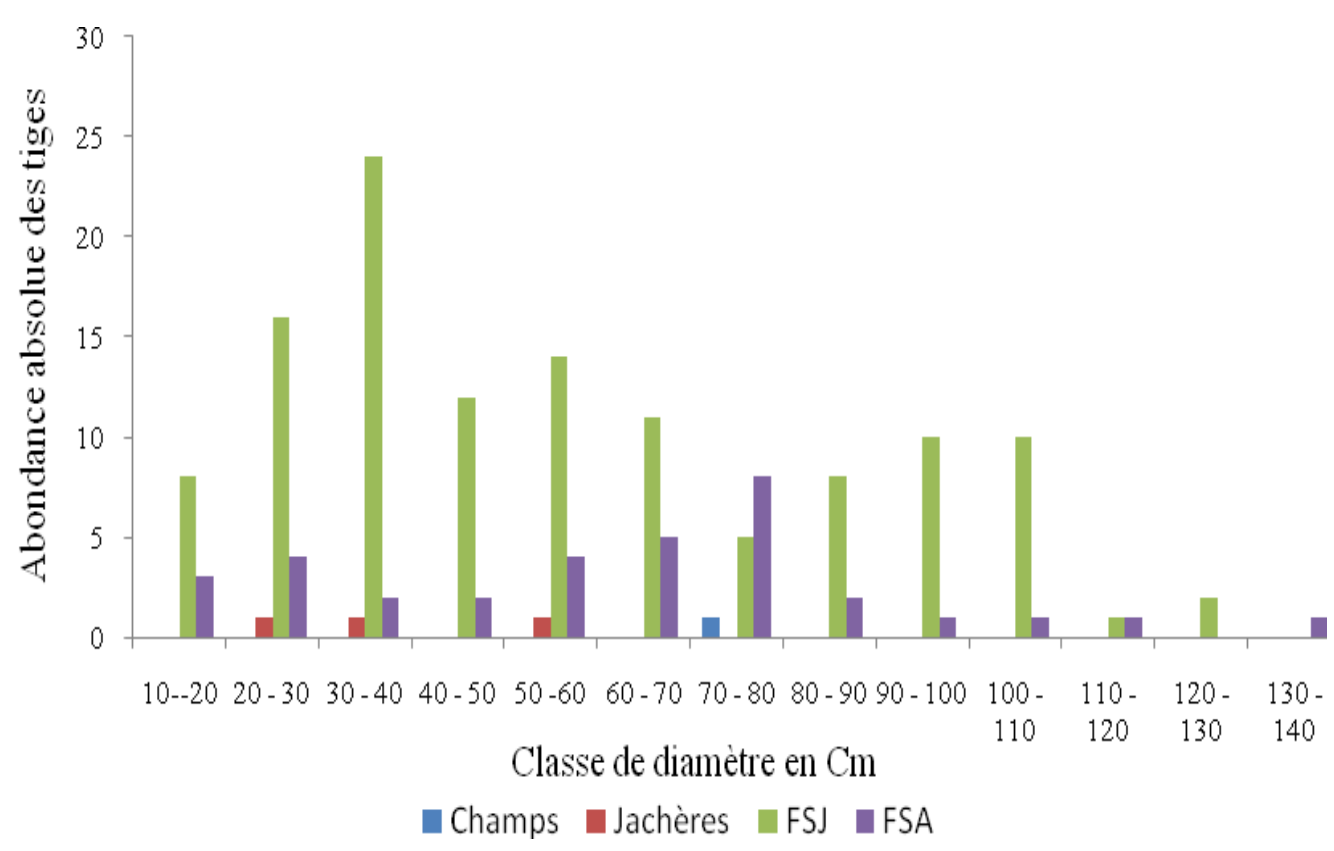

Figure 5: Répartition des individus en classes de diamètre dans les types d'habitat. FSJ : Forêts secondaires jeunes, FSA : Forêts secondaires âgées. 


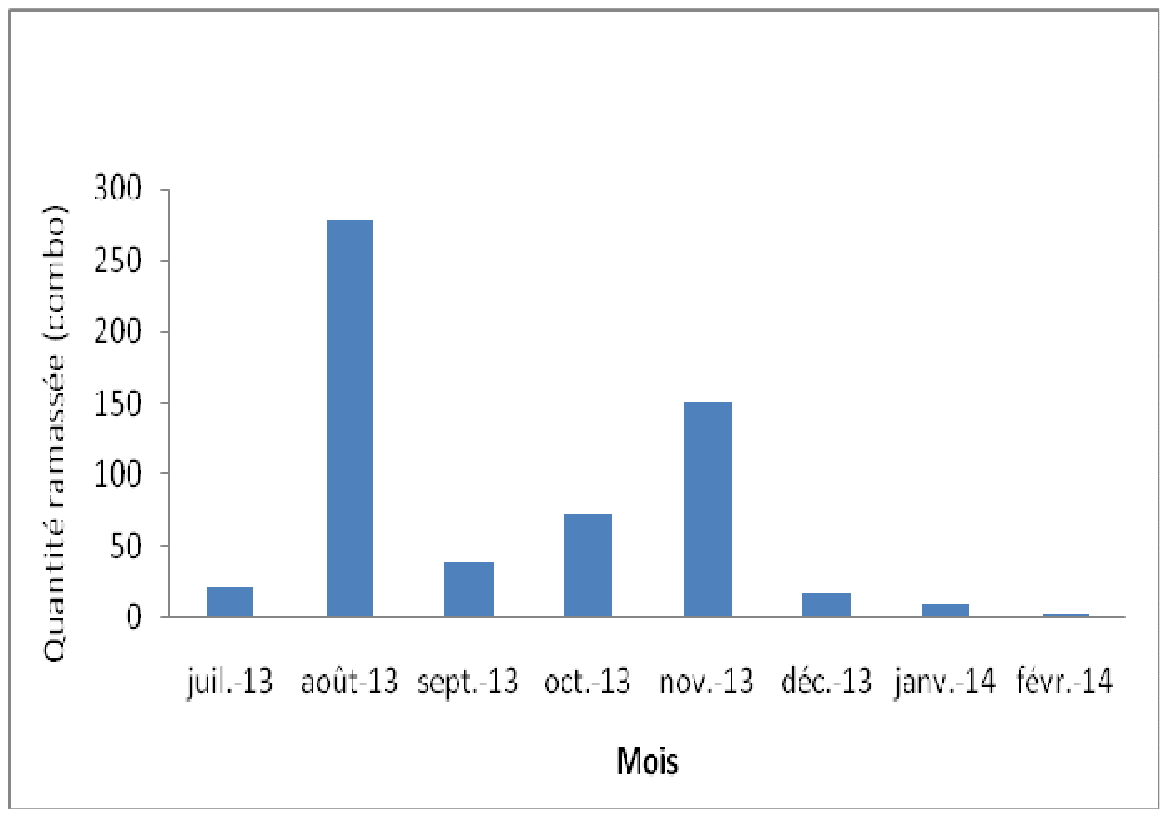

Figure 6: Dynamique de collecte des graines de $P$. macrophylla pendant la période de fructification.

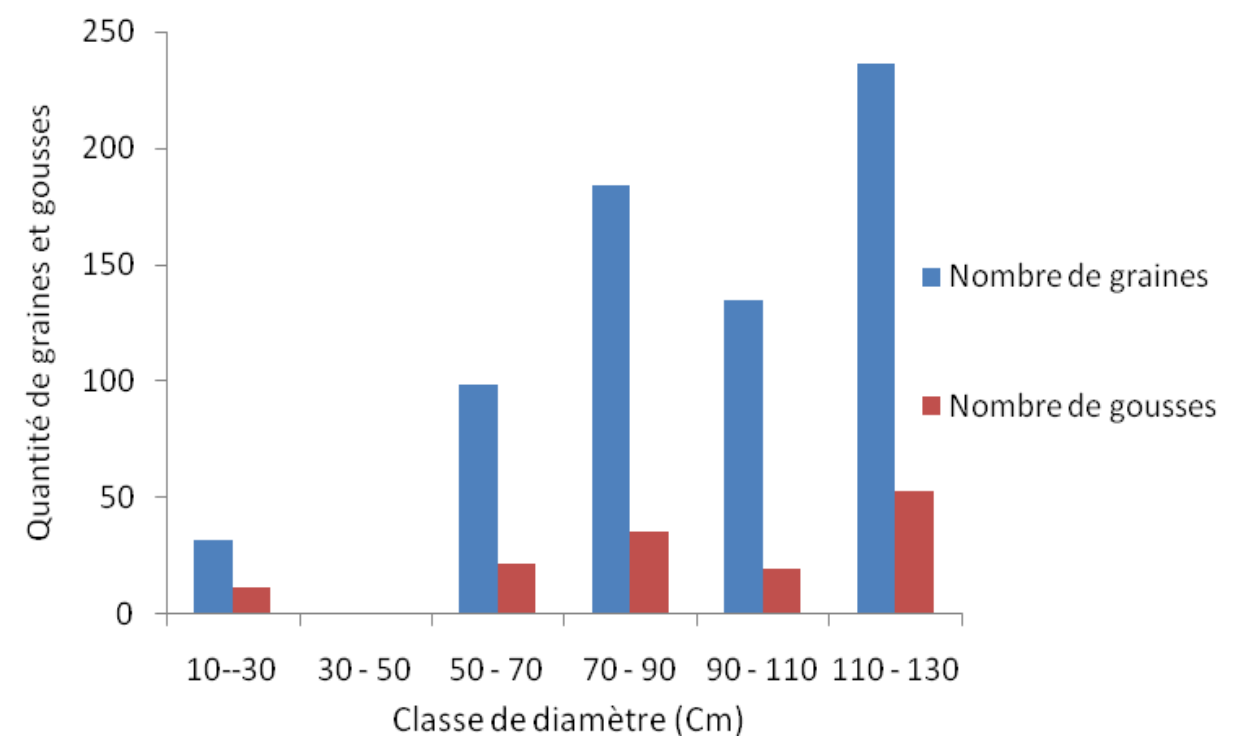

Figure 7 : Répartition par classe de diamètre des tiges adultes des graines et gousses produites. 


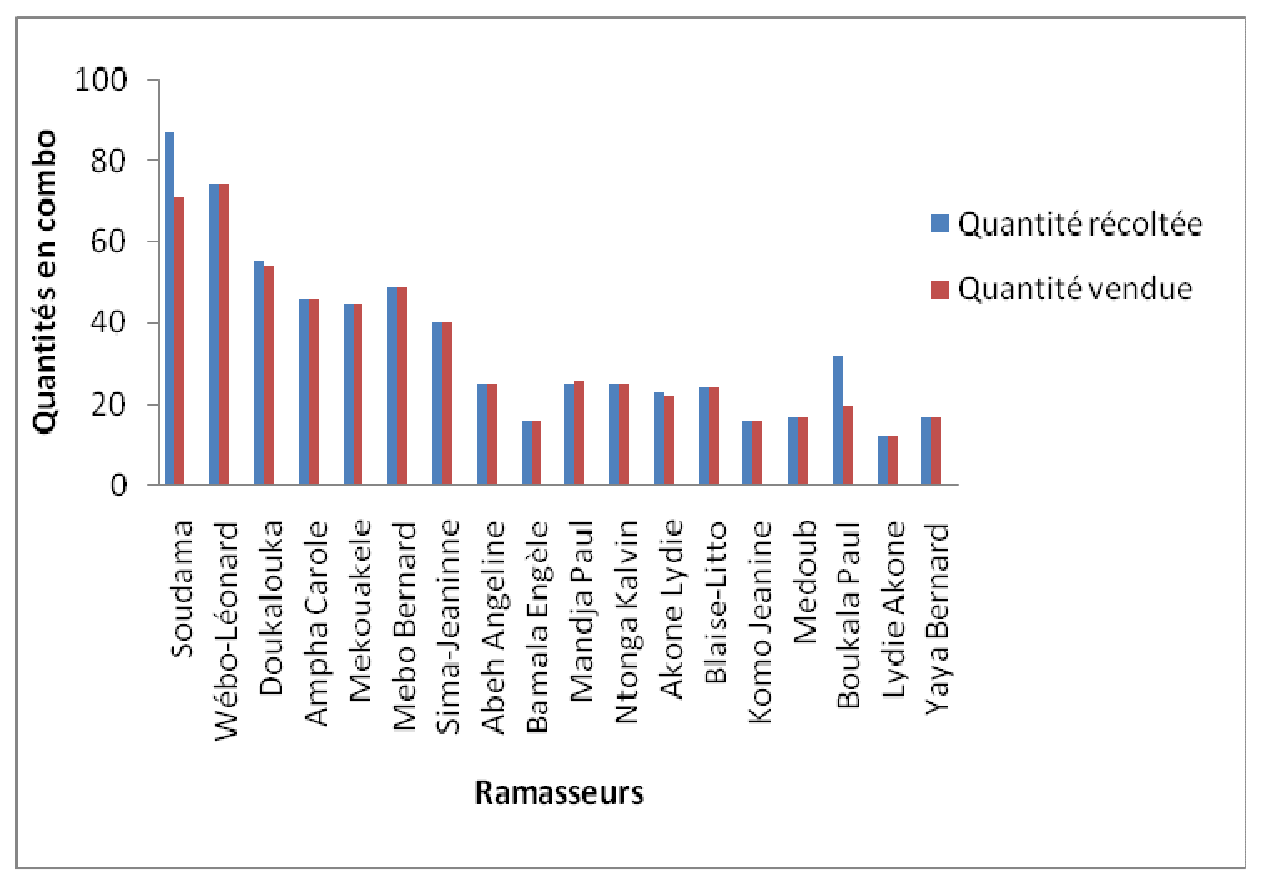

Figure 8 : Comparaison entre les quantités de produit collectées et les quantités vendues.

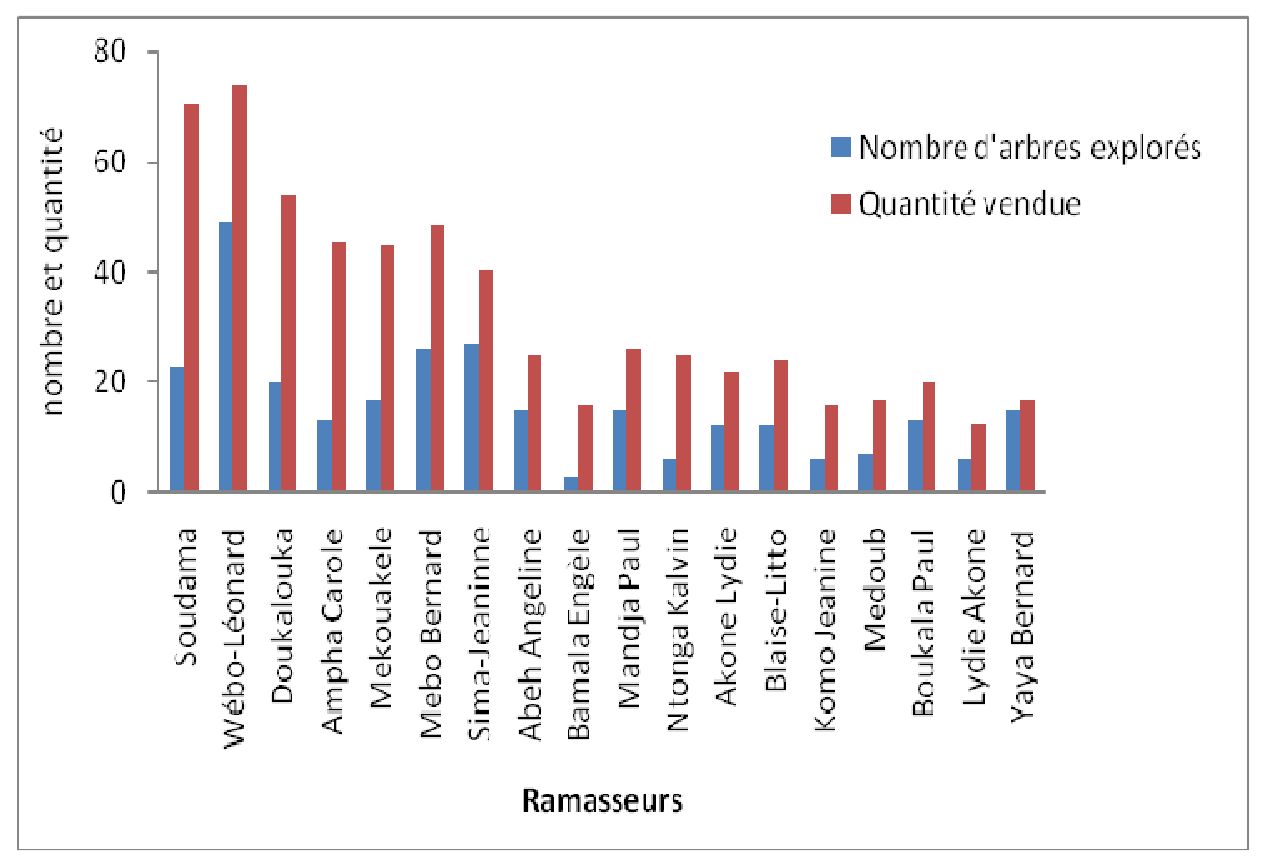

Figure 9: Rapport entre le nombre d'arbres explorés et les quantités collectées et vendues. 
M. MOUAMFON et al. / Int. J. Biol. Chem. Sci. 9(1): 200-216, 2015

Tableau 1 : Paramètres dendrométriques de $P$. macrophylla dans les types d'habitats prospectés.

\begin{tabular}{lccccc}
\hline TH & Nombre de tige & Superficie (ha) & Densité (tige/ha) & $\begin{array}{c}\text { Surface terrière (m²/ha) } \\
\text { Champs }\end{array} \quad 1 \quad \begin{array}{c}\text { Diamètre à hauteur de poitrine moyen } \\
(\mathbf{c m})\end{array}$ \\
Jachères & 3 & 0,31 & 3,23 & 0,17 & 73,85 \\
FSJ & 122 & 1,02 & 2,94 & 0,16 & $39,47 \pm 14$ \\
FSA & 35 & 20,57 & 5,93 & 14,23 & $54,22 \pm 29$ \\
Moyenne & & 10,1 & 3,47 & 4,90 & $60,09 \pm 31$ \\
\multicolumn{2}{r}{ TH : Type d'habitat, FSJ : Forêt secondaire jeune, FSA : Forêt secondaire adulte } & & $56,91 \pm 14$ \\
\hline
\end{tabular}

TH : Type d'habitat, FSJ : Forêt secondaire jeune, FSA : Forêt secondaire adulte

Tableau 2: Abondance des tiges (brins) dans les classes de régénération naturelle.

\begin{tabular}{|c|c|c|c|c|}
\hline \multirow[t]{3}{*}{ TH } & \multicolumn{3}{|c|}{ Classe de hauteur } & \multirow[t]{3}{*}{ Nombre de brins/ha } \\
\hline & \multirow{2}{*}{$\begin{array}{l}\text { Plantule } \\
\text { ]0-100[ }\end{array}$} & \multicolumn{2}{|c|}{ Gaulis } & \\
\hline & & [100 - 200[ & {$[200-400[$} & \\
\hline Jachères & 231 & 56 & 16 & 303 \\
\hline FSJ & 356 & 23 & 8 & 387 \\
\hline FSA & 67 & 46 & 21 & 134 \\
\hline Moyenne (brins/ha) & 218 & 42 & 15 & 824 \\
\hline
\end{tabular}


Tableau 3: Distances de chute des graines et hauteurs des jeunes tiges correspondantes.

\begin{tabular}{lcccc}
\hline & $\begin{array}{c}\text { Distance minimale } \\
(\mathbf{m})\end{array}$ & $\begin{array}{c}\text { Hauteur } \\
\text { plantule }(\mathbf{m})\end{array}$ & $\begin{array}{c}\text { Distance } \\
\text { maximale }(\mathbf{m})\end{array}$ & $\begin{array}{c}\text { Hauteur } \\
\text { plantule }(\mathbf{m})\end{array}$ \\
\hline Jachères & 1,21 & 0,95 & 37,89 & 2,55 \\
FSJ & 1,00 & 0,45 & 40,06 & 0,92 \\
FSA & 1,5 & 0,46 & 38,84 & 0,86 \\
Moyenne & 1,08 & 0,40 & 39,15 & 1,44 \\
Ecart-type & $\pm 0,55$ & $\pm 0,09$ & $\pm 5,82$ & $\pm 0,96$ \\
\hline
\end{tabular}

FSJ : Forêt secondaire jeune, FSA : Forêt secondaire adulte

\section{RESULTATS}

Distribution dans les différents types d'utilisation des terres

La Figure 4 présente la répartition des pieds de $P$. macrophylla inventoriés sur les quatre transects de la forêt communautaire de Payo. Il ressort que l'espèce est inégalement répartie sur les différents transects et présente quelques poches de concentration par endroit.

Analyse comparative de la structure des arbres de $\boldsymbol{P}$. macrophylla dans les différents types d'habitats

Le nombre de tiges varie d'un type d'habitat à l'autre et peut être groupé en deux: le groupe des forêts secondaires jeunes et les forêts secondaires agées et le groupe des jachères et champs vivriers. Dans le premier groupe, les pieds de P. macrophylla sont représentés dans toutes les classes de diamètre jusqu'à la classe [110-120[cm sans distinction de type d'habitat. Par contre dans les classes [120-130[ et [130-140[, les pieds représentés appartiennent respectivement aux forêts secondaires jeunes et forêts secondaires agées. Le nombre de tiges dans les FSJ est toujours supérieur à celui des FSA dans les différentes classes de diamètre (Figure 5). C'est dans la classe $[70-80[\mathrm{~cm}$ qu'il est recensé le plus grand nombre de pieds de $P$. macrophylla dans les FSA. Alors que le plus grand nombre d'individus se trouve dans la classe [30$40[\mathrm{~cm}$ pour les FSJ. Le deuxième groupe constitué des jachères et champs est très faiblement représenté en termes d'effectifs. Il est noté un nombre constant d'individus uniquement dans les classes moyennes $25 \mathrm{~cm}$ à $55 \mathrm{~cm}$, à l'exception de la classe moyenne $45 \mathrm{~cm}$ dans les jachères (Figure 5).

Au total, 161 tiges ont été recensées le long de quatre transects soit une densité moyenne de 5,35 $\pm 1,38$ tiges/ ha. Le nombre de tiges varie d'un type d'habitat à l'autre, mais pas forcément en fonction des superficies parcourues. Les forêts secondaires jeunes (FSJ) et forêts secondaires agées (FSA) ont les plus grandes superficies soit respectivement 20,57 ha (avec 122 individus) et 10,01 ha ( 35 individus), ensuite les jachères et les champs avec respectivement 1,02 ha (3 individus) et 0,31 ha ( 1 individu) (Tableau 1 ).

$\mathrm{La}$ surface terrière traduit le recouvrement de $P$. macrophylla. La surface terrière total est de $19,46 \mathrm{~m}^{2} / \mathrm{ha}$, pour un diamètre moyen de 56,91 $\pm 14 \mathrm{~cm}$ des tiges inventoriées. Les valeurs de la surface terrière, pour tous les types d'habitat varient de 14,23 $\mathrm{m}^{2} / \mathrm{ha}$ pour les FSJ à $0,16 \mathrm{~m}^{2} / \mathrm{ha}$ pour les jachères, avec des valeurs intermédiaires de 4,90 et 0,17 respectivement pour les FSJ et champs (Tableau 1). La plus grande valeur des FSJ correspond au plus grand nombre de tiges (50). Par contre, la différence des valeurs observées entre les champs (une tige) et les jachères (3 tiges), est liée aux valeurs des diamètres plus grands dans les champs que dans les jachères. Globalement, elle ne varie pas en fonction du nombre d'individus mais 
de leur diamètre et de la surface échantillonnée.

Evaluation du potentiel de régénération de P. macrophylla

\section{Classe de régénération naturelle}

Le Tableau 2 présente, pour les différents habitats, la densité des brins constitués des plantules et gaulis dans les classes de régénération. Pour les brins de hauteur comprise entre 0 et $400 \mathrm{~cm}$, on a recensé un total de 824 brins sur une superficie totale de 1,04 ha; soit une densité qui varie de 387 à 134 brins/ha respectivement pour les forêts secondaires agées (FSA) et les forêts secondaires jeunes (FSJ), en passant par la jachère avec une densité de 303 brins/ha.

Les valeurs moyennes de la densité, pour les différents types d'habitat, sont de 218 brins/ha pour les plantules, de 42 et 15 brins/ha respectivement pour les gaulis de hauteur comprise entre [100 - 200 [cm et [200 - 400[ cm. Dans la population d'arbres de la jachère, on enregistre 231 plantules, soit une proportion de $28 \%$ du total des brins; 56 gaulis entre [100 et 200[ $\mathrm{cm}$ de hauteur, soit $6,8 \% ; 16$ gaulis entre [200 et 400[ $\mathrm{cm}$ de hauteur, soit $1,9 \%$. Pour les forêts secondaires jeunes, on enregistre au total 356 plantules, soit une proportion de $43,2 \%$ du total des brins, 23 gaulis entre [100 et 200[ $\mathrm{cm}$ de hauteur, soit 2,8\%; 8 gaulis entre [200 et 400[ $\mathrm{cm}$ de hauteur, soit $0,1 \%$. Au sein de la population d'arbres des forêts secondaires adultes, on dénombre 67 plantules, soit $8,13 \%$; 46 gaulis entre [100 et 200[ $\mathrm{cm}$ de hauteur, soit $5,6 \% ; 21$ gaulis entre [200 et 400 [ $\mathrm{cm}$ de hauteur, soit une représentation de $2,5 \%$.

\section{Détermination de l'indice de régénération}

La valeur de l'indice de régénération naturelle $(I R N)$ a été déterminée par le rapport de la proportion des jeunes individus (Dhp < $10 \mathrm{~cm}$ ) sur celle des adultes (DHP $\geq 10 \mathrm{~cm}$ ). L'étude montre les variations de valeur de l'indice de régénération naturelle (rapport entre le nombre d'individu de diamètre $<10$ $\mathrm{cm}$ sur le nombre d'individu de diamètre $\geq$ $10 \mathrm{~cm})$ de $P$. macrophylla suivant les types d'habitat. Il révèle un indice de régénération moyen de 4,8 pour la zone d'étude. La valeur est plus élevée dans la jachère qui correspond à 24,5, puis les FSJ avec 3,9 et enfin les FSA avec la valeur 2,5.

Potentiel de dissémination sous le semencier

Les jeunes tiges ont tendance à se développer de façon grégaire et en général pas très loin des semenciers. Les valeurs moyennes de chute varient de $1,08 \pm 0,55 \mathrm{~m}$ pour la distance minimale et de $39,15 \pm 5,82$ $\mathrm{m}$ pour la distance maximale et suivant les types d'utilisation des terres (Tableau 3). On constate que la distance maximale de chute est plus élevée dans les FSJ (40,06 m), puis les FSA $(38,84 \mathrm{~m})$ et enfin la jachère $(37,89 \mathrm{~m})$. Alors que la distance minimale est plus élevée dans les FSA $(1,5 \mathrm{~m})$, puis la jachère $(1,21 \mathrm{~m})$ et enfin les FSJ $(1,0 \mathrm{~m})$. Les valeurs moyennes des hauteurs des jeunes tiges repérées aux distances moyennes varient de manière significative de $1,44 \pm 0,96 \mathrm{~m}$ pour la distance maximale et de 40,33 $\pm 0,09 \mathrm{~m}$ pour la distance minimale. Les valeurs des hauteurs des plantules décroissent d'une manière générale des jachères aux FSA en passant par les FSJ pour les distances minimales et inversement pour les distances maximales. On constate que les hauteurs des jeunes tiges augmentent quand on s'éloigne des pieds de l'arbre. En général, les distances et les hauteurs respectives des jeunes individus varient légèrement d'un type d'habitat à l'autre.

\section{Evaluation de la production fruitière de $\boldsymbol{P}$. macrophylla \\ Poids moyen des gousses et graines}

Le poids des gousses varie entre $359 \mathrm{~g}$ pour la classe de longueur [41-50[ $\mathrm{cm}$ et $779 \mathrm{~g}$ pour la classe de longueur [80 et 90[ cm. La 
masse moyenne d'une gousse est de $499 \pm$ $141 \mathrm{~g}$.

Treize assiettes ou «Combo » (unité de mesure locale qui est une assiette de contenance liquide de 2 litres) après décompte, contient 1417 graines. Le poids d'une assiette varie de $2057 \mathrm{~g}$ pour l'assiette contenant 96 grosses graines à 2254 g pour l'assiette contenant 136 petites graines mûres avec une moyenne de 2155,5 g. Le poids moyen de la graine fraîche étant égal à 19,98 $\pm 1,4 \mathrm{~g}$. Les mesures avec trois repétitions faites sur 100 graines ont montré une dominance des graines de la classe de poids [10-20[ avec 55 graines. 95\% de graines ont un poids compris entre 10 et 30 grammes.

\section{Production et valeur des graines de $\boldsymbol{P}$. macrophylla}

Production et disponibilité des graines

Les fruits sont présents de juin à janvier ou février, avec un maximum en novembre et décembre. La chute des graines qui dure trois à quatre mois suivant la saison de fructification, présente deux pics de production (août et novembre). La dynamique de collecte au courant de la période de fructification traduit le rythme de chute des graines dans la forêt (Figure 6).

Sur 35 pieds d'arbres et après une période de fructification, 5157 graines ont été comptées correspondant à 1063 gousses. Le nombre de graines et de gousses varie d'une tige à l'autre (12 à 613 pour les graines et de 3 à 143 pour les gousses). Les valeurs moyennes du nombre de graines et de gousses sont respectivement de 147,34 \pm 93 et 29,3 \pm 17. Les quantités des produits (graines et gousses) de l'arbre varient considérablement d'un arbre à l'autre, ce qui justifie la valeur élevée de l'écart-type égale à \pm 17 pour les graines et \pm 93 pour les gousses.

Il y a une relation entre graines, gousses produites et le diamètre de l'arbre. Le nombre de graines et gousses augmente avec le diamètre de tiges. L'allure générale des histogrammes des deux variables est la même et se présente sous la forme d'un « $\mathrm{S}$ » couché (Figure 7). Les deux variables sont fortement liées pour la production. Cette structure croit de manière linéaire avec un coefficient de variation $R^{2}=0,812$, des plus petites aux plus grandes classes de diamètre. La structure caractérise une production régulière et croissante qui n'est pas forcément fonction de la taille ou de l'âge de l'arbre.

\section{Estimation de la valeur de production des graines de P. macrophylla}

La commercialisation des graines est prometteuse dans la mesure où presque toute la collecte est toujours vendue, les quantités recoltées et celles vendues étant presque identiques avec un pourcentage de vente de 98\%. Ces quantités sont estimées en unité de mesure locales des graines (Figure 8).

Il est aussi observé que les quantités collectées sont liées au nombre d'arbres explorés par les ramasseurs; plus le nombre d'arbres est élevé mieux la collecte est consistante (Figure 9). Dans le village Payo et ses environs, les populations vont à des distances comprises entre $0,5 \mathrm{~km}$ et $7 \mathrm{~km}$ afin de collecter les graines. 07 campements de collecte sur 14 sont situés à plus de $5 \mathrm{~km}$ des villages.

Ces données suggèrent que la collecte de $P$. macrophylla est avant tout une entreprise commerciale, l'autoconsommation jouant un rôle mineur ou quasi inexistant. Une somme de 632625 frs CFA a été générée par la vente de ces graines, dans le village Payo par les personnes suivies pendant la campagne de collecte de l'année 2013. Pris individuellement, les gains aucours de cette période, sont compris entre 300 (pour celui qui n'a vendu qu'une seule fois) et 55850 frs CFA (pour un ramasseur regulier).

Le calcul de la densité a permis d'estimer le nombre de tiges à l'hectare dans la forêt communautaire de Payo. Après la projection et prenant en compte la densité (D) des arbres de $P$. macrophylla calculée $(5,35$ 
tiges /ha), la superficie (S) de la forêt comunautaire en question (1 600 ha), la production moyenne (p) de 35 tiges evaluées (5 157 graines), la contenance (Q) moyenne du combo (unité de mesure) à 109 graines, et le prix moyen $(\mathrm{F}) \mathrm{d}$ 'achat du combo à 500 frs CFA, nous estimons la production de la forêt communautaire de Payo $\left(\mathrm{P}_{\mathrm{fc}}\right)$ et la valeur des graines $\left(\mathrm{V}_{\mathrm{fc}}\right)$ au moyen des formules suivantes :

$\mathrm{P}_{\mathrm{fc}}=\frac{\mathrm{D} x \mathrm{~S} x \mathrm{p}}{35} \quad$ et $\quad \mathrm{V}_{\mathrm{fc}}=\frac{\mathrm{P}_{\mathrm{fc}}}{\mathrm{Q}} \times \mathrm{F}$.

Nous obtenons ainsi : $\mathrm{P}_{\mathrm{fc}}$ égale à 1261255 graines equivalent à 11571 combo pour une valeur totale $V_{\text {fc }}$ de 5785572 Frs CFA. Cette somme est la valeur estimée de ce que peut rapporter la forêt communautaire de Payo au cours d'une campagne de collecte.

Les produits récoltés en forêt, en l'occurrence les graines, sont collectées au village (Payo) par les paysans et relais puis vendues dans les marchés nationaux (Lomié, Abong-bang, Yaoundé, Douala) aux collecteurs et grossistes camerounais et étrangers qui exportent enfin vers le Nigéria où le marché est très important et plus rentable. Les prix d'achat ou vente au niveau des villages ne sont pas standardisés et varient considérablement de 300 à 1000 frs CFA le combo (assiette de contenance de 2 litres), en fonction de la période, de la quantité collectée et des besoins des populations qui mènent l'activité de ramassage.

\section{DISCUSSION}

\section{Distribution de $\boldsymbol{P}$. macrophylla}

La présente étude a montré que les pieds de $P$. macrophylla sont inégalement distribués dans les différents types d'utilisation des terres. Ce résultat montre que $P$. macrophylla est une espèce végétale des forêts secondaires des régions tropicales comme l'indique les travaux de Oboh (2007) et de Gwamashi (2009).
La densité de 5,35 tiges/ha obtenue, témoigne d'une bonne représentation de l'espèce dans la localité de Payo. Dans la zone de Djoum au sud Cameroun, l'espèce est abondante dans les forêts, les jachères, et les jardins de case et présente une densité moyenne de 10,74 tiges /ha, deux fois supérieure à celle de Lomié (Mandeng, 2009). L'espèce est donc plus abondante à Djoum. $P$. macrophylla doit aussi sa présence significative dans la forêt communautaire de Payo par une très forte occupation de la zone par les «Baka » qui sont fortement rattachés à leur tradition. Ils conservent l'espèce dans ce milieu pour des pratiques socioculturelles. Les gousses sont utilisées contre les mauvais sorts et les graines utilisées contre les morsures des serpents. En Côte d'Ivoire, dans la région de Taï, l'espèce est aussi utilisée pour lutter contre les mauvais esprits (Koné et al., 2008). L'espèce est conservée pour ses valeurs alimentaires et médicinales :l'arbre sert de lieu de collecte du miel, les graines, les feuilles et les écorces pour les valeurs alimentaires et thérapeutiques. Dans la zone forstière humide d'Afrique, les graines sont preparées et consommées dans plusieurs ménages (Oboh, 2007). L'homme developpe donc une affinité culturelle et alimentaire avec l'espèce.

Les faibles densités dans les champs et jachères sont dues aux actions anthropiques à travers la déforestation et l'utilisation des feux de brousse qui dégradent considérablement le potentiel disponible de l'espèce. Cette faible densité peut être attibuée aussi à la faible représentation de ces types d'habitat dans les 04 transects. Ces transects étant perpendiculaires à la route qui est l'axe du village et les champs et jachères étant concentrés aux environs des habitations.

\section{Evaluation du potentiel de régénération de P. macrophylla}

L'évaluation du potentiel de régénération naturelle dans la zone montre 
que $P$. macrophylla dans la forêt communautaire de Payo est équilibré (IRN = 4,8). Havyarimana (2009) et Hakizimana et al. (2011), rapportent que l'état de régénération équilibrée est confirmé par les valeurs de l'indice de régénération naturelle qui sont toutes supérieures à l'unité. P. macrophylla a un bon indice de régénération d'où une forte capacité de renouvellement; or Eyog et al. (2006) et Mandeng (2009) préconisaient déjà la domestication de l'espèce pour sa conservation. La valeur de l'indice de régénération varie suivant les systèmes d'utilisation des terres. Cette valeur diminue au fur et à mésure que l'on passe des champs aux FSA en passant par jachères et FSJ. Cette variation peut être due aux actions anthropiques et l'éclairement dont l'intensité diminue de la jachère aux formations secondaires jeunes et adultes où la canopée a une forte influence dans le sous-bois.

La dissémination des jeunes individus de $P$. macrophylla autour des sémenciers isolés, met en évidence l'existence des jeunes tiges installées entre 0,45 et $46,38 \mathrm{~m}$. On observe aussi qu'au fur et à mesure que l'on part des classes de diamètres inférieurs (Dhp < $10 \mathrm{~cm}$ ) pour les classes de diamètres supérieurs (Dhp $\geq 10 \mathrm{~cm}$ ) dans les différents TH étudiés, le nombre d'individus diminue. Ce qui est en cohérence avec les travaux de Jansen et Forget (2001), au cours desquels la dissémination des graines de $C$. surinamensis en Guyane s'effectue en grande majorité à des distances inférieures à 10 mètres des limites de la couronne des arbres parents. Plus on s'éloigne du pied de l'arbre mature, plus le nombre des jeunes individus et des graines tombées de $P$. macrophylla diminue et plus la hauteur des jeunes individus augmente. Ces différences de valeurs peuvent être dues à la taille de l'arbre, aux structures des différentes formations végétales explorées et aux agents de dissémination tels que les rongeurs et les humains.
Production et disponibilité des graines de $P$. macrophylla

Comme précédemment énoncé, l'exploitation actuelle de P.macrophylla qui est pratiquée de manière locale dans la forêt communautaire de Payo est fortement influencée par les techniques utilisées. Les populations locales doivent braver d'énormes difficultés pour se procurer des quantités considérables de graines pour la commercialisation. La production estimée (5 157 graines pour un total de 35 arbres) au cours de la période d'étude n'est toujours pas égale à la quantité ramassée. Les périodes de fructification et de production des pieds matures ainsi que la liberation des graines qui est fonction du dégré d'ensoleillement sont aussi des facteurs qui reduisent la production disponible et les quantités mises en vente, c'est aussi le cas de Carapa sp. (Nyiramana, 2012). Actuellement, la plupart des collecteurs achètent ces graines accompagnées d'autres PFNL déjà bien connus et plusieurs paysans après les activités champêtres ramassent ces graines quand ils vont à la recherche des amandes d'Irginvigia gabonensis. Une production importante et disponible générerait un revenu plus considérable et concurrencer d'autres PFNL après les produits agricoles. Dans le même ordre d'idées, Lescuyer (2010) affirme que les revenus issus de la cueillette sont nettement inférieurs à ceux de l'agriculture. Ceux qui ramassent les graines dans la localité évoquent la consommation des graines par les rongeurs comme une difficulté.

\section{Valeur des graines de $\boldsymbol{P}$. macrophylla}

Avec $98 \%$ des collectes vendues, la commercialisation des graines bien que moins attractive que celle des PFNL bien connus est une activité profitable surtout que les paysans n'ont aucun intrant ou traitement à faire. $80 \%$ des personnes interrogées en Repuplique du Congo tire profit du commerce des PFNL (Loubelo, 2012). Les produits peuvent aussi générer des revenus importants 
aux populations locales s'ils sont exploités et gérés de manière continue et rationnelle. La valeur des produits vendus au village est fixée par l'acheteur et ces prix parfois dérisoires poussent au découragement des ramasseurs qui continuent l'activité parce qu'ils sont dans le besoin d'argent pour leur ménage. Loubelo (2012) explique cette situation en ce sens que la contribution des PFNL au revenu des ménages, par rapport à celle d'autres activités, est très variable. Plusieurs facteurs justifient cette variabilité, notamment : l'activité principale du ménage, l'origine du PFNL et l'étape à laquelle intervient le ménage dans la filière.

\section{Conclusion}

Les populations de $P$. macrophylla sont abondantes dans la forêt communautaire de Payo. Elles sont plus fréquentes en forêts secondaires jeunes qu'en forêts secondaires adultes, mais moins fréquentes dans les jachères et champs. L'espèce présente une bonne surface de couverture. Les plantules sont plus nombreuses dans les forêts secondaires que dans les jachères et champs.

$P$. macrophylla génère théoriquement au bout d'une année et sur une superficie de 1 600 ha, près de cinq million sept cent quatre vingt cinq mille cinq cent soixante douze (5 785572 ) Frs CFA dont onze mille huit cent trente six (11 836) \$US. Avec l'intérêt croissant que les commerçants accordent à ces graines, l'espèce peut aussi contribuer à relever le niveau de vie des populations de Payo et valoriser la forêt communautaire.

$\mathrm{Au}$ regard de ce qui précède, l'exploitation actuelle des produits de $P$. macrophylla n'exerce pas encore une forte pression sur la survie de cette espèce. Il ressort donc de cette étude que le potentiel écologique et productif de $P$. macrophylla pour l'instant dans la forêt communautaire de Payo témoigne d'une bonne distribution, d'une capacité productive et d'un potentiel de régénération important. Ceci assure une bonne pérennité de l'espèce pour la conservation de la biodiversité au Cameroun. Actuellement, le Nigeria est la seule destination connue des graines collectées par les principaux acheteurs qui partent de la zone de Lomié et d'autres localités du Cameroun. L'amélioration du circuit de commercialisation et la valorisation au Cameroun des produits derivés donnerait une plus-value au graines très prisées au Nigeria, pays voisin.

\section{REFERENCES}

Betti JL, Lejoly J. 2010. Contribution à la connaissance des plantes médicinales de la Réserve de Biosphère du Dja au Cameroun: plantes utilisées dans le traitement des maux de dos. Int. J. Biol. Chem. Sci. 4(1): 193-200.

Ernst C, Verhegghen A, Mayaux P, Hansen M, Defourny P. 2012. Cartographie du couvert forestier et des changements du couvert forestier en Afrique centrale. In Les Forêts du Bassin du Congo : Etat des Forêst 2010, de Wasseige C, de Marcken $\mathrm{P}$, Bayol N, Hiol Hiol F, Mayaux Ph, Desclée B, Nasi R, Billand A, Defourny $\mathrm{P}$, Eba'a Atyi R (eds). Office des Publications de l'Union Européenne : Luxembourg; 23-42.

Eyog M, Ndoye O, Kengue J, Awono A. 2006. Les Fruitiers Forestiers Comestibles $d u$ Cameroun. Center for International Forestry Research. IPGRI: Yaoundé; 220.

Fokou SI. 2008. Evaluation de quelques produits forestiers non ligneux de la région de Lomié à l'Est du Cameroun. Mémoire d'études supérieures spécialisées (DESS) en sciences forestières, Université de Yaoundé 1, Yaoundé, p. 56.

Forget P-M. 1996. Removal of seeds of Carapa procera (Meliaceae) by rodents and their fate in rainforest in French Guiana. J. Trop. Ecol., 12: 751-761. 
Guedje NM, Bigombe LP. 2010. Harvesting bark from Non Timber Forest Products : the case of Garcinia lucida(Clusiaceae). In Systematics and Conservation of African Plants, van der Burgt X, van der Maesen J, Onana JM (eds). Royal Botanic Gardens: Kew, UK; 351-358.

Guedje NM, Fokunang CN, Jiofack TRB, Dongmo FR. 2010. Opportunités d'une exploitation soutenue des plantes médicinales dans l'aménagement forestier. Int. J. Biol. Chem. Sci., 4(4): 1346-1372.

Guedje NM. 2002. La gestion des populations d'arbres comme outil pour une exploitation durable des Produits Forestiers Non Ligneux : L'exemple de Garcinia lucida (Sud-Cameroun). Thèse de doctorat $\mathrm{PhD}$, Université Libre de Bruxelles, Bruxelles, p. 266.

Gwamashi ET. 2009. Inventaire des espèces ligneuses locales pour le reboisement à des fins énergétiques. Mémoire Master, Université de Kinshasa. http://www. memoireonline.com.

Hakizimana P, Bangirinama F, Havyarimana F, Habonimana B, Bogaert J. 2011. Analyse de l'effet de la structure spatiale des arbres sur la régénération naturelle de la forêt claire de Rumonge au Burundi. Bull. Sci. Inst. Natl. Environ. Conserv. Nat. 9: 46-52.

Hall P, Bawa K. 1993. Methods to assess the impact of extraction of non timber tropical forest products on plant population. Economic Botany, 47(3): 234247.

Havyarimana F. 2009. Impact de la distribution spatiale des espèces arborescentes sur la diversité végétale dans la réserve naturelle forestière de Bururi (Burundi). Thèse de Doctorat, Université Libre de Bruxelles, Bruxelles, p. 55.
Jansen PA, Forget PM 2001. Scatter-hoarding by rodents and tree regeneration in French Guiana. In Nouragues: Dynamics and Plant-Animal Interactions in a Neotropical Rainforest, Bongers FP, Charles-Dominique, Forget PM, Théry M (Eds). Kluwer Academic: Dordrecht, The Netherlands; 275-288.

Koné I, Lambert JE, Refisch J, Bakayoko A. 2008. Primate seed dispersal and its potential role in maintaining useful tree species in the Taï region, Côte-d'Ivoire: implications for the conservation of forest fragments. Tropical Conservation Science, 1(3): 293-306.

Lescuyer G. 2010. Importance économique des produits forestiers non ligneux dans quelques villages du Sud-Cameroun. Bois et Forêts des Tropiques, 304(2): 15-24.

Loubelo E. 2012. Impact des Produits Forestiers Non Ligneux (PFNL) sur l'économie des ménages et la sécurité alimentaire : Cas de la République du Congo. Thèse de Doctorat PhD. Université Rennes 2, Rennes, p. 260.

Mandeng NMO. 2009. Evaluation des potentialités de domestication de l'ébaye (Pentaclethra macrophylla Benth.) par les Pygmées Baka de l'Arrondissement de Djoum, Région du Sud du Cameroun. Mémoire d'Ingénierie de conception des Eaux, Forêts et Chasse, Université de Dschang, Dschang, p. 64.

Mouamfon M, Guedje MN, Zapfack L, Lejoly J. 2010. Exploitation des essences commerciales dans les forêts communautaires de Kompia et Kabilone (Est-Cameroun): Difficultés et perspectives. In Systematics and Conservation of African Plants, van der Burgt X, van der Maesen J, Onana JM. (eds.). Royal Botanic Gardens: Kew, UK; 501-508.

Ngueguim JR. 2013. Productivité et diversité floristique des ligneux en forêt dense d'Afrique tropicale humide du Cameroun: 
sites de Mangombé, Bidou et Campo. Thèse de PhD, Muséum National d'Histoire Naturelle de Paris, Paris, p.195.

Nyiramana A. 2012. Production de fruits, dissémination et devenir des graines de Carapa grandiflora sprague (Meliacée) dans le Parc National de Nyungwe, Rwanda. Thèse de Doctorat. Muséum National d'Histoire Naturelle, Paris, Paris, p. 226.

Oboh G. 2007. Pentaclethra macrophylla Benth. In Vegetable Oils/Oléagineux, Van der Vossen, Kamilo MGS (éds). PROTA 14 : Wageningen, Hollande; [En ligne]. Consulté le 07 août à 14 h $03 \mathrm{mn}$. Disponible sur http //www.database. prota.org/PROTA html/
Priso RJ, Nnanga JF, Etame J, Din N, Amougou A, 2011. Les produits forestiers non ligneux d'origine végétale valeur et importances. Journal of Applied Bio-Sciences, 40(1): 2715-2726.

Tsobeng A, Asaah E, Makueti J, TchoundjeuZ, Van Damme P. 2013. Propagation of Pentaclethra macrophylla Benth (Fabaceae) through seed and rooting of leafy stem cuttings. International Journal of Agronomy and Agriculture Research, 3(12): 10-20.

Vivien J, Faure JJ, 1996. Fruitiers Sauvages d'Afrique (Espèces du Cameroun). Editions Nguila-Kérou : Clohars CanoetFrance ; 416. 